\title{
Manifestaciones orales asociadas a SARS-COV-2: Revisión de la literatura
}

\author{
Oral manifestations associated to SARS-COV-2: A literature review \\ Manifestações orais associadas ao SARS-COV-2: Revisão da literatura
}

\author{
Natalia Sandoval-Gómez' (iD) 0000-0003-3120-4076 \\ Tamara Needham-Torres' ${ }^{1}$ iD 0000-0002-9644-572X \\ Gisella Vásquez-Canales ${ }^{1}$ (D) 0000-0001-6401-5170 \\ Ana María Salazar-Roa ${ }^{2}$ (D) 0000-0003-3536-9310
}

\section{Resumen}

Objetivo: Identificar las principales manifestaciones y describir su ubicación en la cavidad oral en pacientes COVID-19.

Métodos: Se utilizaron las bases de datos PubMed, Medline, LILACS, LIVIVO, Web of Science y SciELO; utilizando los términos de búsqueda oral mucosa, oral mucosa lesion, oral manifestations, COVID-19 y SARS-CoV-2. Se eliminaron duplicados, luego se realizó preselección de artículos, y finalmente se aplicaron los criterios de inclusión y exclusión.

Resultados: Se seleccionaron 47 publicaciones, encontrando manifestaciones orales en pacientes COVID-19 tales como alteración en gusto, xerostomía, úlceras, vesículas, entre otras; ubicándose en diferentes áreas de la mucosa oral.

Conclusiones: Se necesitan más estudios para vislumbrar la posible etiopatogenia a nivel oral del SARS-CoV-2. Además, se destaca el rol del odontólogo en el equipo multidisciplinario y en la teleconsulta.

Palabras clave: COVID-19, manifestación oral, mucosa oral.

$1 \quad$ Facultad de Odontología Universidad de Concepción, Chile.n475sg@gmail.com

2 Departamento de patología y diagnóstico, Facultad de Odontología, Universidad de Concepción, Chile.

Fecha de recibido: 15/1/2021 - Fecha de aceptado: 22/3/2021 


\section{Abstract}

Aim: Identify the main oral manifestations and describe their location in the oral cavity, in patients with COVID-19.

Methods: The literature search was developed in the following databases: PubMed, Medline, LILACS, LIVIVO, Web of Science and SciELO; combining the following words: oral mucosa, oral mucosa lesion, oral manifestations, COVID-19 and SARSCoV-2. Duplicated articles were eliminated, and then a preselection of articles was made. Finally, inclusion and exclusion criteria were applied.

Results: 47 articles were included in this study. The main oral manifestations in patients with COVID-19 are taste disorders, xerostomia, ulcers, vesicles, and others; with location in different areas of the oral mucosa. Conclusion: More studies are needed to conceive the possible oral pathogenesis of SARS-CoV-2. Moreover, the role of the dentist in the multidisciplinary team and teleconsultation are highlighted.

Keywords: COVID-19, oral manifestation, oral mucosa.

\section{Introducción}

El surgimiento de la nueva enfermedad infecciosa: Coronavirus 2019 (COVID-19), cuya etiología es el Síndrome Respiratorio Agudo Severo Coronavirus 2 (SARS-CoV-2), ha tenido un impacto masivo en todo el mundo; a causa del modo de propagación de la infección, las disfunciones respiratorias severas resultantes y el número de muertes a nivel glo-

\section{Resumo}

Objetivo: Identificar as principais manifestações e descobrir sua ubiquação na cavidade oral em pacientes com COVID-19.

Método: Foram utilizadas as bases de dados PubMed, Medline, LILACS, LIVIVO, Web of Science e SciELO; utilizando os termos de pesquisa oral mucosa, oral mucosa lesion, oral manifestations, COVID-19 e SARS-CoV-2. Duplicadas foram removidas, depois uma pré-seleção de artigos foi feita, e finalmente os critérios de inclusão e exclusão foram aplicados.

Resultados: Foram selecionadas 47 publicações, encontrando manifestações orais em pacientes com COVID-19, tais como alteraçóes no paladar, xerostomia, ulceraçôes, vesículas, entre outros; localizando-as em diferentes áreas da mucosa oral.

Conclusão: São precisos mais estudos pra vislumbrar a possível etiopatogenia a nível oral do SARS-CoV-2. Ademais, destaca-se o role do odontólogo na equipe multidisciplinar e na tele consulta.

Palavras-chave: COVID-19, Manifestação oral, mucosa oral. bal, comprometiendo de manera significativa la calidad de vida de las personas ${ }^{(1)}$. Desde su aparición en Wuhan, China; esta enfermedad ha alcanzado una alta tasa de infectividad con proyecciones aproximadamente en todo el mundo de 47 millones de casos, y una tasa de mortalidad que oscila entre el 3\% y el 12\%, superando el millón de fallecidos ${ }^{(1-3)}$. 
El origen específico de esta pandemia no se comprende en su totalidad. Aunque no se tiene seguridad de la forma en que el ser humano adquirió esta enfermedad, se estudia que fue a partir de un animal; siendo los murciélagos los principales seres vivos salvajes que poseen reservorios de este tipo de virus, produciéndose un salto viral entre este animal y un ser humano en Wuhan, China durante noviembre del $2019^{(2,4)}$.

En cuanto a la forma o rutas de transmisión de COVID-19 entre seres humanos; ésta se da principalmente por gotas de saliva expulsadas al hablar ${ }^{(1,2,5-9)}$ o estornudar dentro de una distancia de 2 metros, o al estar en contacto con superficies expuestas ${ }^{(2,6-9)}$. El $50 \%$ de las transmisiones ocurren como consecuencia de la exposición a personas asintomáticas ${ }^{(2)}$, siendo importante destacar que el paciente puede transmitir la infección hasta 2 semanas después de haberse recuperado de los síntomas de la enfermedad ${ }^{(4)}$.

Las investigaciones actuales muestran que el virus SARS-CoV-2 invade las células humanas a través del receptor de la Enzima Convertidora

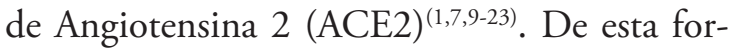
ma, las células con distribución del receptor ACE2 pueden convertirse en células hospedadoras del virus y causar una respuesta inflamatoria en órganos y tejidos anexos, destacándose entre ellos, la cavidad oral ${ }^{(1,7,8,10,12,14,16-19,21-28)}$.

Esta revisión de la literatura tiene como objetivo identificar las principales manifestaciones a nivel oral en pacientes positivos a COVID-19 y describir su ubicación dentro de la cavidad oral.

\section{Materiales y métodos}

Para el desarrollo de esta revisión de la literatura se establecieron las siguientes etapas: definición del tema, definición de los objetivos, especificación de los criterios de inclusión y exclusión, elaboración del plan de trabajo para realizar la búsqueda de la literatura, selección y recuperación de los estudios que cumplen los criterios, extracción de los datos para realizar un análisis y síntesis de los resultados obteniendo finalmente la formulación de la conclusión.

En un periodo del 9 de Octubre 2020 al 30 Diciembre 2020, se realizó una búsqueda en la base de datos electrónicas: PubMed, Medline, LILACS, LIVIVO, Web of Science y SciELO.

Los términos de búsqueda definidos para realizar la búsqueda en la base de datos fueron: oral mucosa, oral mucosa lesion, oral manifestations, COVID-19 y SARS-CoV-2, acompañadas de los operadores booleanos OR y AND. Obteniéndose: ((COVID-19) OR (SARS-CoV-2)) AND (mucosa oral)), ((COVID-19) OR (SARS-CoV-2)) AND (mucosa oral lesion)) y ((COVID-19)OR (SARS-CoV-2)) AND (oral manifestations)), se procedió a leer título y abstract para determinar posible idoneidad, luego de esta selección se leyeron los textos completos para identificar si cumplían los criterios de inclusión y exclusión.

Entre los criterios de inclusión se consideraron artículos dentro de los últimos 2 años, que incorporan los términos de búsqueda y que estuvieran tanto en espańol como inglés.

Entre los criterios de exclusión quedaron fuera aquellos estudios realizados en animales, enfocados al tratamiento de pacientes infectados con el virus SARS-CoV-2, pacientes covid-19 sin manifestaciones a nivel oral o aquellos relacionados con medidas de prevención y/o bioseguridad que no se ajustan a los objetivos planteados. 
Figura 1. Diagrama de flujo del proceso de búsqueda, revisión y selección de artículos

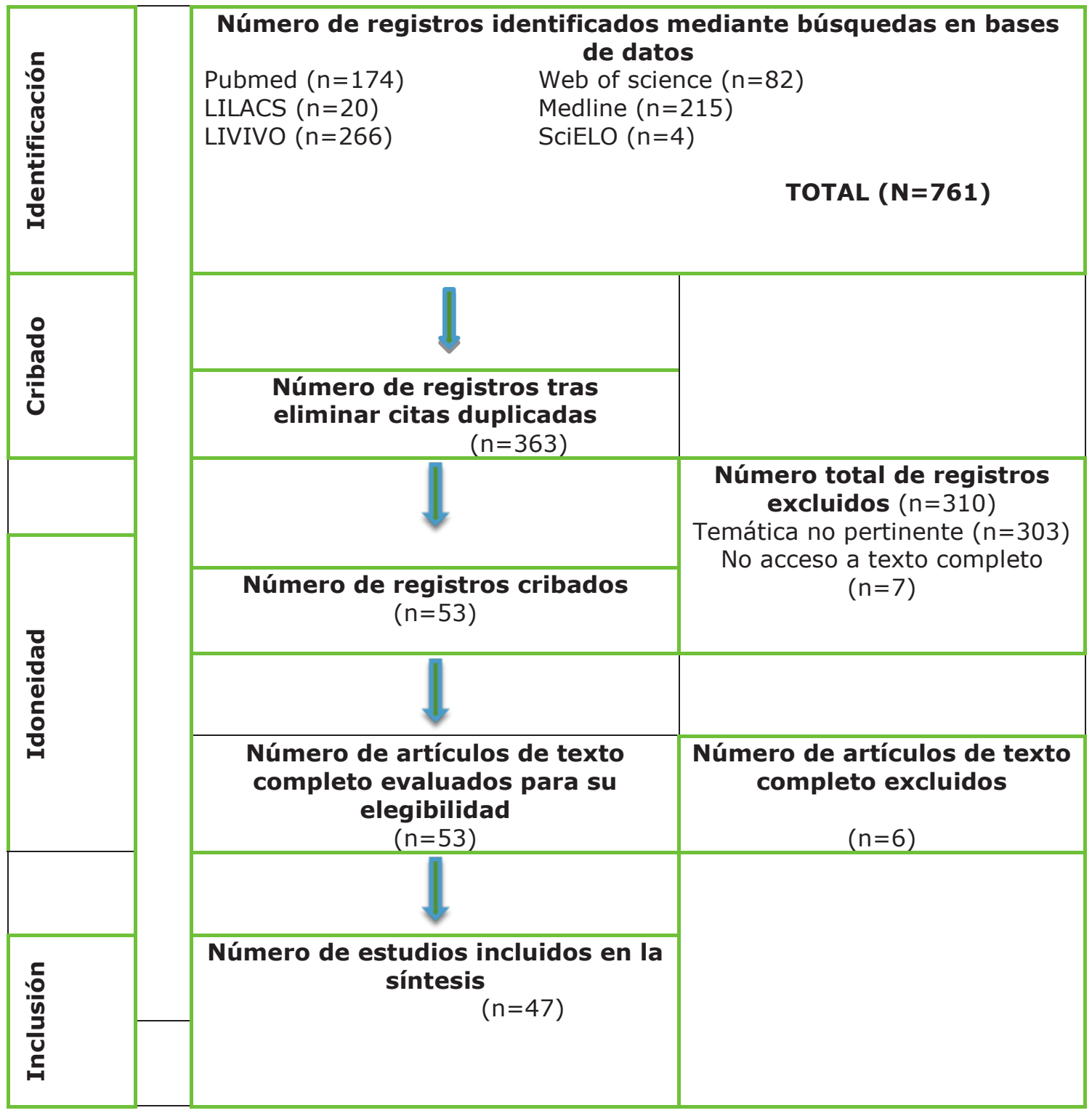

\section{Desarrollo}

Del total de artículos encontrados se eliminaron los duplicados y se seleccionaron estudios que cumplieran con los criterios de inclusión y exclusión, seleccionando finalmente: 47 artículos, de los cuales 9 son reportes de caso, 1 caso clínico con revisión bibliográfica narrativa, 2 revisión sistemática, 3 revisión crítica, 9 revisión narrativa, 4 estudios observacionales y 19 cartas al editor; además se incorporó información de 1 artículo e información de 2 sitios web para la introducción.

De los casos reportados en la literatura, se han encontrado lesiones bastante heterogéneas entre pacientes, e incluso en un mismo paciente $\mathrm{e}^{(10-13)}$ describiendo distintas presentaciones, 
localizaciones y $\operatorname{tamaños}^{(5,10,12,29)}$. Las manifestaciones a nivel oral se han presentado en pacientes COVID-19 sin antecedentes médicos de relevancia, describiéndose sanos ${ }^{(1,5,12,13,18,30-33)}$ y también en pacientes que presentan enfermedades de base ${ }^{(1,7,10,12,13,16,18,23,27-30,33-41)}$.
Las manifestaciones mencionadas con mayor frecuencia son la alteración del gusto y úlceras, mientras que las menos mencionadas son pústulas, glositis y depapilación lingual. A continuación (tabla 1) se describen las principales manifestaciones encontradas:

Tabla 1: Manifestaciones Orales reportadas en pacientes COVID-19

\begin{tabular}{|c|c|c|}
\hline Manifestación Oral & Tipo de Publicación & Referencias \\
\hline Alteración en gusto & $\begin{array}{c}5 \text { Reportes de Caso } \\
2 \text { Revisión Sistemática } \\
7 \text { Revisión Narrativa } \\
2 \text { Revisión crítica } \\
10 \text { Cartas al editor } \\
3 \text { Estudios observacionales } \\
1 \text { Caso Clínico con revisión bibliográfica narrativa }\end{array}$ & $\begin{array}{c}30 \text { Referencias } \\
(1,6-12,14,16-19,21-24,26,29,30,33,36,38,40-46)\end{array}$ \\
\hline Sensación de ardor & $\begin{array}{c}1 \text { Reportes de Caso } \\
3 \text { Revisión Narrativa } \\
3 \text { Cartas al editor } \\
2 \text { Estudio observacional } \\
1 \text { Caso Clínico con revisión bibliográfica narrativa }\end{array}$ & $\begin{array}{c}10 \text { Referencias } \\
(6,8,12,18,27,36,39,41,44,47)\end{array}$ \\
\hline Xerostomía & $\begin{array}{l}2 \text { Reportes de Caso } \\
5 \text { Revisión Narrativa } \\
1 \text { Revisión Crítica } \\
2 \text { Cartas al editor } \\
2 \text { Estudio observacional }\end{array}$ & $\begin{array}{c}12 \text { Referencias } \\
(5,6,8-10,16-18,24,29,36,47)\end{array}$ \\
\hline Halitosis & $\begin{array}{c}3 \text { Revisión Narrativa } \\
2 \text { Cartas al editor }\end{array}$ & $\begin{array}{c}5 \text { Referencias } \\
(16,17,25,27,48)\end{array}$ \\
\hline Eritema & $\begin{array}{c}4 \text { Reportes de Caso } \\
1 \text { Revisión Narrativa } \\
1 \text { Revisión Sistemática } \\
3 \text { Revisión Crítica } \\
4 \text { Cartas al editor } \\
1 \text { Estudio observacional } \\
1 \text { Caso Clínico con revisión bibliográfica narrativa }\end{array}$ & $\begin{array}{c}15 \text { Referencias } \\
(1,9-13,19,20,27,28,30,31,41,46,49)\end{array}$ \\
\hline Petequias & $\begin{array}{c}2 \text { Reportes de Caso } \\
4 \text { Revisión Narrativa } \\
1 \text { Revisión Sistemática } \\
2 \text { Revisión Crítica } \\
3 \text { Cartas al editor } \\
1 \text { Caso Clínico con revisión bibliográfica narrativa }\end{array}$ & $\begin{array}{c}13 \text { Referencias } \\
(1,5,9,11,13,15,17,18,20,26,27,31,41)\end{array}$ \\
\hline
\end{tabular}




\begin{tabular}{|c|c|c|}
\hline Manifestación Oral & Tipo de Publicación & Referencias \\
\hline Máculas & $\begin{array}{c}2 \text { Reportes de Caso } \\
1 \text { Revisión Narrativa } \\
1 \text { Revisión Sistemática } \\
2 \text { Revisión Crítica } \\
3 \text { Cartas al editor } \\
1 \text { Estudio observacional } \\
1 \text { Caso Clínico con revisión bibliográfica narrativa }\end{array}$ & $\begin{array}{l}11 \text { Referencias } \\
(10-13,17,19,2,2,26,29,41,47)\end{array}$ \\
\hline Vesículo-Bullosas/Ampollas & $\begin{array}{c}3 \text { Reportes de Caso } \\
5 \text { Revisión Narrativa } \\
1 \text { Revisión Sistemática } \\
3 \text { Revisión Crítica } \\
3 \text { Cartas al editor } \\
1 \text { Estudio observacional } \\
1 \text { Caso Clínico con revisión bibliográfica narrativa }\end{array}$ & $\begin{array}{c}17 \text { Referencias } \\
(8,9,11,12,15-20,23,26,6,28,30,37,41,49)\end{array}$ \\
\hline Pústula & $\begin{array}{l}1 \text { Reportes de Caso } \\
1 \text { Revisión Narrativa } \\
2 \text { Cartas al editor }\end{array}$ & $\begin{array}{c}4 \text { Referencias } \\
(13,27,31,32)\end{array}$ \\
\hline Úlceras & $\begin{array}{c}6 \text { Reportes de Caso } \\
6 \text { Revisión Narrativa } \\
1 \text { Revisión Sistemática } \\
3 \text { Revisión Crítica } \\
11 \text { Cartas al editor } \\
2 \text { Estudio observacional } \\
1 \text { Caso Clínico con revisión bibliográfica narrativa }\end{array}$ & $\begin{array}{c}30 \text { Referencias } \\
(1,5,6,6-13,15-20,23,25-30,33-55,38,41,44-46)\end{array}$ \\
\hline Gingivitis descamativa & $\begin{array}{l}2 \text { Reportes de Caso } \\
3 \text { Revisión Narrativa } \\
1 \text { Revisión Sistemática } \\
2 \text { Revisión Crítica } \\
1 \text { Cartas al editor }\end{array}$ & $\begin{array}{l}9 \text { Referencias } \\
(9,11,12,15,18,19,25,2,3,30)\end{array}$ \\
\hline $\begin{array}{l}\text { Infección de glándulas } \\
\text { salivales }\end{array}$ & $\begin{array}{c}3 \text { Revisión Narrativa } \\
2 \text { Revisión Crítica } \\
2 \text { Cartas al editor } \\
1 \text { Caso Clínico con revisión bibliográfica narrativa }\end{array}$ & $\begin{array}{l}8 \text { Referencias } \\
(8,19-21,24,28,41,45)\end{array}$ \\
\hline Depapilación lingual & 2 Cartas al editor & $\underset{(6,44)}{2 \text { Referencias }}$ \\
\hline Glositis & $\begin{array}{l}3 \text { Revisión Narrativa } \\
1 \text { Revisión Crítica } \\
2 \text { Cartas al editor }\end{array}$ & $\begin{array}{c}6 \text { Referencias } \\
(9,16,17,27,39,44)\end{array}$ \\
\hline $\begin{array}{l}\text { Coinfección con } \\
\text { Candidiasis }\end{array}$ & $\begin{array}{c}2 \text { Reportes de Caso } \\
1 \text { Revisión Narrativa } \\
1 \text { Revisión Sistemática } \\
2 \text { Cartas al editor } \\
1 \text { Estudio observacional } \\
1 \text { Caso Clínico con revisión bibliográfica narrativa }\end{array}$ & $\begin{array}{l}8 \text { Referencias } \\
(5,6,610,11,1,7,79,41,46)\end{array}$ \\
\hline
\end{tabular}




\begin{tabular}{|c|c|c|}
\hline Manifestación Oral & Tipo de Publicación & Referencias \\
\hline Lesiones de aspecto herpe- & 4 Reportes de Caso & 14 Referencias \\
\hline tiforme & 3 Revisión Narrativa & $(1,9-11,14,17,18,26,27,30,33,41,42,46)$ \\
\hline & 1 Revisión Sistemática \\
\hline & 1 Revisión Crítica \\
\hline & 3 Cartas al editor \\
\hline
\end{tabular}

En cuanto a su localización, ésta resulta inespecífica, encontrándose principalmente a nivel de tan lesiones en mucosa bucal, encía y orofaringe paladar, lengua, dorso principalmente, y labio, (Tabla 2).

Tabla 2: Localización de las manifestaciones orales reportadas en pacientes COVID-19

\begin{tabular}{|c|c|c|}
\hline Localización & Tipo de Publicación & Referencias \\
\hline Paladar & $\begin{array}{c}3 \text { Reportes de Caso } \\
4 \text { Revislón Narrativa } \\
1 \text { Revisión Sistemática } \\
2 \text { Revisión Crítica } \\
10 \text { Cartas al editor } \\
1 \text { Estudio observacional } \\
1 \text { Caso Clínico con revisión bibliográfica narrativa }\end{array}$ & $\begin{array}{c}22 \text { Referencias } \\
(1,6,9,11-13,15,17,18,20,23,26-31,35,38,39,41,43)\end{array}$ \\
\hline Lengua & $\begin{array}{c}5 \text { Reportes de Caso } \\
2 \text { Revisión Narrativa } \\
1 \text { Revisión Sistemática } \\
2 \text { Revisión Crítica } \\
11 \text { Cartas al editor } \\
2 \text { Estudio observacional } \\
1 \text { Caso Clínico con revisión bibliográfica narrativa }\end{array}$ & $\begin{array}{c}24 \text { Referencias } \\
(11,5,6,9-12,17,20,23,26-29,32,33,35,38,39,41,44-46,49)\end{array}$ \\
\hline Labio & $\begin{array}{c}6 \text { Reportes de Caso } \\
4 \text { Revisión Narrativa } \\
1 \text { Revisión Sistemática } \\
2 \text { Revisión Crítica } \\
7 \text { Cartas al editor } \\
2 \text { Estudio observacional } \\
1 \text { Caso Clínico con revisión bibliográfica narrativa }\end{array}$ & $\begin{array}{c}23 \text { Referencias } \\
(1,5,6,9,11,13,15,17,18,20,23,26-30,34,37,38,41,42,46,49)\end{array}$ \\
\hline Mucosa bucal & $\begin{array}{c}2 \text { Reportes de Caso } \\
2 \text { Revisión Narrativa } \\
1 \text { Revisión Sistemática } \\
1 \text { Revisión Crítica } \\
3 \text { Cartas al editor } \\
1 \text { Estudio observacional }\end{array}$ & $\begin{array}{c}10 \text { Referencias } \\
(1,9,11,15,27,28,29,37,46,49)\end{array}$ \\
\hline
\end{tabular}




\begin{tabular}{|c|c|c|}
\hline Localización & Tipo de Publicación & Referencias \\
\hline Encía & $\begin{array}{c}1 \text { Reportes de Caso } \\
3 \text { Revisión Narrativa } \\
1 \text { Revisión Sistemática } \\
1 \text { Revisión Crítica } \\
5 \text { Cartas al editor } \\
1 \text { Caso Clínico con revisión bibliográfica narrativa }\end{array}$ & 12 Referencias $(5,6,9,911,13,15,18,26-26-28,41,48)$ \\
\hline Orofaringe & $\begin{array}{c}2 \text { Reportes de Caso } \\
2 \text { Revisión Narrativa } \\
1 \text { Revisión Sistemática } \\
2 \text { Cartas al editor } \\
1 \text { Estudio observacional }\end{array}$ & $\begin{array}{l}8 \text { Referencias } \\
(5,10,11,13,18,23,27,31)\end{array}$ \\
\hline
\end{tabular}

\section{Discusión}

Si bien se han reportado lesiones en mucosa oral en pacientes infectados con el virus SARSCOV-2, se cree que la causa no sería el virus propiamente tal, sino que las lesiones serían secundarias a los fármacos ${ }^{(5,7,9-11,15,17,18,21,26,28,30,32,36,39,41 \text {, }}$ 43,50), administrados para el tratamiento, o debido compromiso y deterioro del sistema inmune $^{(1,6,9-12,15-17,26,27,33,34,36,38,41,45,50)}$, lo cual también trae consigo infecciones oportunis$\operatorname{tas}^{(5,6,9,-11,15-18,25-28,33,36,39,41,42,45,48,50)}$. Lo más reportado son coinfecciones de Cándida albicans ${ }^{(5,6,10,}$ $11,17,39,41) y / u$ otros virus ${ }^{(1,9,10,16,17,27,33,41-43,45,46,50)}$. Pese a estas suposiciones, algunos de los estudios revisados señalan la teoría de un efecto vascular y trombótico en la mucosa oral generado por el virus SARS-CoV-2 $2^{(6,9,12,15,17,23,27,29,34)}$. De igual manera, las lesiones de la mucosa oral podrían ser desencadenadas por factores como estrés ${ }^{(5,8,18,22,25,26,32,37)}$.

Es fundamental recalcar la importancia del receptor de ACE2 (Enzima Convertidora de Angiotensina 2), este está distribuído en la membrana celular de diversos órganos y tejidos, tales como el tracto respiratorio, sistema nervioso, sistema digestivo y músculo esquelético ${ }^{(1,9,13,23,24,27)}$. Dentro de la cavidad bucal se presenta en células epiteliales de mucosa oral $^{(1,7,8,12,16-19,21-27)}$ y gingival ${ }^{(7,14,21,23,24)}$, destaca su presencia en células epiteliales de lengua $^{(1,7-11,14,15,17,18,21,24,25,27,33,36)}$ y glándulas salivales $^{(1,9-12,16-20,24,27,36)}$, debido a la alta expresión a nivel lingual ${ }^{(8,9,11,14,15,18,19,21,22,24,25,27,33)}$, se describe la lengua como un órgano susceptible $e^{(14,19,25,33)}$. Algunos autores destacan que las glándulas salivales ${ }^{(6,8,10,21,24,36)}$ y sacos periodontales ${ }^{(9,18,25,36,44)}$ podrían actuar como reservorio del virus.

Este sería el principal receptor del virus SARS$\mathrm{CoV}-2$, generando que las células con distribución de este receptor desencadenen reacciones inflamatorias en los órganos y tejidos asocia$\operatorname{dos}^{(1,9,10,13)}$, es por ello que a nivel lingual, diversos autores señalan que esta interacción podría ser responsable del desarrollo de trastornos en el gusto en pacientes con la enfermedad COVID-19 $9^{(1,8-11,15-19,22,24)}$. Además se ha descrito que la interacción entre el virus y este receptor altera la función de los queratinocitos orales y el revestimiento epitelial de los conductos de las glándulas salivales tras la infección ${ }^{(1)}$. Adicionalmente, la saliva y la secreción nasal podrían ser fluidos con alta carga viral viéndose involucrados en el desarrollo de estas alteraciones orales descritas ${ }^{(9,12)}$.

Algo en lo que coinciden múltiples autores es la necesidad de realizar estudios adicionales e investigación exhaustiva ${ }^{(1,5,9-16,23,24,26,28-31,34-36,42,43,50)}$ para conocer los mecanismos patogénicos del SARS-CoV-2 sobre los tejidos orales, y así determinar si las lesiones en mucosa oral tienen relación directa o indirecta con la progresión del COVID-19 ${ }^{(1)}$. 


\section{Conclusión}

Las manifestaciones orales en pacientes COVID-19 han sido reportadas en múltiples lugares de la cavidad oral y tienen presentaciones bastante heterogéneas, dada la actualidad del tema, la prevalencia de estas manifestaciones aún es desconocida, pues los casos reportados en la literatura son pocos en comparación con el número de pacientes infectados, y la mayoría de estos casos carece de exámenes complementarios, como biopsias o exámenes hematoló- gicos, que ayuden a vislumbrar el real agente etiológico. Todo ello deja interrogantes abiertas con respecto a la posible etiopatogenia o factores que podrían estar influyendo en el desarrollo de estas lesiones, incentivando a realizar más estudios al respecto.

Finalmente es importante reconocer la participación del odontólogo tanto en el equipo de salud como a través de la telemedicina, con el fin de realizar un cauteloso examen bucal para lograr diagnóstico, tratamiento y control del dolor de las lesiones descritas.

\section{Referencias}

1. Brandão TB, Gueiros LA, Melo TS, et al. Oral lesions in patients with SARS-CoV-2 infection: could the oral cavity be a target organ? Oral Surg Oral Med Oral Pathol Oral Radiol. 2020;S22124403(20)31119-6.

2. World Health Organization. Coronavirus disease (COVID-19) pandemic. Situations Reports. EPIWIN Updates (no 28 What we know about COVID-19). 2020 [Accessed November 05, 2020] Available from: https://www.who.int/emergencies/diseases/novel-coronavirus-2019

3. Worldmeter [Homepage on the internet] COVID-19 Coronavirus Pandemic. 2020 [Accessed October 21, 2020]. Available from: https://www.worldometers.info/coronavirus/.

4. Ortiz-Prado E, Simbaña-Rivera K, Gómez-Barreno L, et al. Clinical, molecular, and epidemiological characterization of the SARS-CoV-2 virus and the Coronavirus Disease 2019 (COVID-19), a comprehensive literature review. Diagn Microbiol Infect Dis. 2020;98(1):115094.

5. Corchuelo J, Ulloa FC. Oral manifestations in a patient with a history of asymptomatic COVID-19: Case report. Int J Infect Dis. 2020;100:154-157.

6. Díaz Rodríguez M, Jimenez Romera A, Villarroel M. Oral manifestations associated with COVID-19. Oral Dis. 2020; doi: 10.1111/odi.13555.

7. Orellana-Centeno JE, Morales-Castillo V, Guerrero SRN. Generalidades, manejos, cuidados y manifestaciones clínicas del SARS-CoV-2. Rev ADM. 2020;77(3):153-155.

8. Pant, B. COVID-19: Oral manifestations, impact and lessons for the future. Orthodontic Journal of Nepal. 2020; 10(2), 52-54.

9. Parra-Sanabria EA, Bermúdez-Bermúdez M, Peña-Vega CP, Rueda-Jiménez A. Manifestaciones orales y maxilofaciales asociadas a la COVID-19. Revisión de la literatura. Acta Odont Col. 2020; 10(Supl. COVID-19): 60-80.

10. Amorim Dos Santos J, Normando AGC, Carvalho da Silva RL, De Paula RM, Cembranel AC, SantosSilva AR, Guerra ENS. Oral mucosal lesions in a COVID-19 patient: New signs or secondary manifestations? Int J Infect Dis. 2020;97:326-328.

11. Amorim Dos Santos J, Normando AGC, Carvalho da Silva RL, Acevedo AC, De Luca Canto G, Sugaya N, Santos-Silva AR, Guerra ENS. Oral Manifestations in Patients with COVID-19: A Living Systematic Review. J Dent Res. 2020;11:22034520957289.

12. Cruz Tapia RO, Peraza Labrador AJ, Guimaraes DM, Matos Valdez LH. Oral mucosal lesions in patients with SARS-CoV-2 infection. Report of four cases. Are they a true sign of COVID-19 disease? Spec Care Dentist. 2020;1-6. 
13. Ciccarese G, Drago F, Boatti M, Porro A, Muzic SI, Parodi A. Oral erosions and petechiae during SARS-CoV-2 infection. J Med Virol. 2020;10.1002/jmv.26221.

14. Cornejo-Ovalle Marco, Espinoza-Santander Iris. COVID-19 y manifestaciones orales. Int. J. Odontostomat. 2020;14( 4 ):538-539.

15. Maciel PP, Martelli Júnior H, Martelli DRB, Machado RA, Andrade PV, Perez DEC, et al. COVID-19 pandemic: oral repercussions and its possible impact on oral health. Pesqui Bras Odontopediatria Clín Integr. 2020; 20(supp1):e0138.

16. Pedrosa MS, Sipert CR, Nogueira FN. Salivary glands, saliva and oral findings in COVID-19 infection. Pesqui Bras Odontopediatria Clín Integr. 2020; 20(supp1):e0104.

17. Nemeth-Kohanszky María Eugenia, Matus-Abásolo Carolina Paz, Carrasco-Soto Rolando Rafael. Manifestaciones Orales de la Infección por COVID-19. Int. J. Odontostomat. 2020; 14(4):555-560.

18. Gutiérrez Flores R, Zambrano Rodríguez G. Implicaciones bucales por COVID-19. Revisión de tema. Odontol Sanmarquina.2020;23(4):419-23.

19. Capocasale G, Nocini R, Faccioni P, Donadello D, Bertossi D, Albanese M, Zotti F. How to deal with coronavirus disease 2019: A comprehensive narrative review about oral involvement of the disease. Clin Exp Dent Res. 2020;10.1002/cre2.332.

20. Halboub E, Al-Maweri SA, Alanazi RH, Qaid NM, Abdulrab S. Orofacial manifestations of COVID-19: a brief review of the published literature. Braz Oral Res 2020;34:e124.

21. Odeh ND, Babkair H, Abu-Hammad S, Borzangy S, Abu-Hammad A, Abu-Hammad O. COVID-19: Present and Future Challenges for Dental Practice. Int J Environ Res Public Health. 2020;17(9):3151.

22. Abalo-Lojo JM, Pouso-Diz JM, Gonzalez F. Taste and Smell Dysfunction in COVID-19 Patients. Annals of Otology, Rhinology \& Laryngology. 2020; 129(10):1041-1042.

23. Katz J,Yue S. Increased odds ratio for COVID 19 in patients with recurrent aphthous stomatitis. J Oral Pathol Med 2020;00:1-4.

24. Pastrian G. Presencia y expresión del receptor ACE2 (Target de SARS-CoV-2) en tejidos humanos y cavidad oral.Posibles rutas de infección en órganos orales. Int. J. Odontostomat. 2020;14(4):501-507.

25. Riad A, Kassem I, Hockova B, Badrah M, Klugar M. Halitosis in COVID-19 patients. Spec Care Dentist. 2020;1-3.

26. de Sousa FACG, Paradella TC. Considerations on oral manifestations of COVID-19. J Med Virol. 2021;93:667-668.

27. Iranmanesh B, Khalili M, Amiri R, Zartab H, Aflatoonian M. Oral manifestations of COVID -19 disease: A review article. Dermatologic Therapy. 2020;e14578.

28. Riad A, Klugar M, Krsek M. COVID-19-Related Oral Manifestations: Early Disease Features? Oral Dis. 2020;00:1-3.

29. Soares CD, Carvalho RA, Carvalho KA, Carvalho MG, Almeida OP. Letter to Editor: Oral lesions in a patient with COVID-19. Med Oral Patol Oral Cir Bucal. 2020;25(4):e563-e564.

30. Martín Carreras-Presas C, Amaro Sánchez J, López-Sánchez AF, Jané-Salas E, Somacarrera Pérez ML. Oral vesiculobullous lesions associated with SARS-CoV-2 infection. Oral Dis. 2020;00:1-3.

31. Cebeci Kahraman F, Çaşkurlu H. Mucosal involvement in a COVID-19-positive patient: A case report. Dermatol Ther. 2020;33(4):e13797.

32. Alzahrani MJ, Moussa MM, Alfaraj D. Acute Generalized Exanthematous Pustulosis After COVID-19 Infection: A Case Report From Saudi Arabia. Cureus. 2020;12(11):e11609.

33. Riad A, Kassem I, Hockova B, Badrah M, Klugar M. Tongue ulcers associated with SARS-CoV-2 infection: A case series. Oral Dis. 2020;00:1-3.

34. Cebeci Kahraman F, Özen T, Elibol T. Lip necrosis in a patient with paroxysmal nocturnal hemoglobinuria: Can it be triggered by COVID-19? J Cosmet Dermatol. 2020;19:3168-3170.

35. Ansari R, Gheitani M, Heidari F, Heidari F. Oral cavity lesions as a manifestation of the novel virus (COVID-19). Oral Dis. 2020. 
36. Sinjari B, D’Ardes D, Santilli M, Rexhepi I, D’Addazio G, Di Carlo P, Chiacchiaretta P, Caputi S, Cipollone F. SARS-CoV-2 and Oral Manifestation: An Observational, Human Study. J Clin Med. 2020;9(10):3218.

37. Bakar Dertlioglu S . Skin rashes in COVID-19: A report of three cases. J Health Sci Med 2020; 3(4): 490-492.

38. Bemquerer LM, de Arruda JAA, Soares MPD, Mesquita RA, Silva TA. The oral cavity cannot be forgotten in the COVID-19 era: is there a connection between dermatological and oral manifestations?, J American Academy Dermatol 2020; S0190-9622(20)33073-5.

39. Riad A, Gad A, Hockova B, Klugar M. Oral Candidiasis in Non-Severe COVID-19 Patients: Call for Antibiotic Stewardship. Oral Surg. 2020; doi: 10.1111/ors.12561.

40. Passarelli PC, Lopez MA, Mastandrea Bonaviri GN, Garcia-Godoy F, D’Addona A. Taste and smell as chemosensory dysfunctions in COVID-19 infection. Am J Dent. 2020;33(3):135-137.

41. Eghbali Zarch R, Hosseinzadeh P.COVID-19 from the perspective of dentists: A case report and brief review of more than 170 cases. Dermatologic Therapy.2021;e14717.

42. Kitakawa D, Oliveira FE, Neves de Castro P, Carvalho LFCS. Short report - Herpes simplex lesion in the lip semimucosa in a COVID-19 patient. Eur Rev Med Pharmacol Sci. 2020;24(17):9151-9153.

43. de Carvalho L, Kitakawa D, Cabral LAG. Oral lesions of herpes zoster in COVID-19 patients or truly associated to the disease? Oral Dis. 2020;00:1-2.

44. Nuno-Gonzalez A, Martin-Carrillo P, Magaletsky K, Martin Rios MD, Herranz Mañas C, Artigas et al. Prevalence of mucocutaneous manifestations in 666 patients with COVID-19 in a field hospital in Spain: oral and palmoplantar findings. Br J Dermatol. 2020;184:158-185.

45. Abu-Hammad S, Dar-Odeh N, Abu-Hammad O. SARS-CoV-2 and oral ulcers: A causative agent or a predisposing factor? Oral Dis. 2020;00:1-2.

46. Mascitti H, Bonsang B, Dinh A, Assan F, Perronne V, Leblanc T, Duran C, Bouchand F, Matt M, Le Gal A, N'guyen Van Thanh J, Lanore A, Jacob L, Kiavue N, Siméon S, Bessis S, de Truchis P, Landowski S, Davido B, Moreau F, Rameix-Welti MA, Gault E, Gaillard JL, Roux AL, Sivadon-Tardy V, Salomon E, El Sayed F, Carlier R, Emile JF, Perronne C, Bourgault-Villada I. Clinical Cutaneous Features of Patients Infected With SARS-CoV-2 Hospitalized for Pneumonia: A Cross-sectional Study. Open Forum Infect Dis. 2020;7(11):ofaa394.

47. Biadsee A, Biadsee A, Kassem F, Dagan O, Masarwa S, Ormianer Z. Olfactory and Oral Manifestations of COVID-19: Sex-Related Symptoms-A Potential Pathway to Early Diagnosis. Otolaryngol Head Neck Surg. 2020;163(4):722-728.

48. Patel J, Woolley J. Necrotizing periodontal disease: Oral manifestation of COVID-19. Oral Dis. 2020;00:1-2.

49. Aghazadeh N, Homayouni M, Sartori-Valinotti JC. Oral vesicles and acral erythema: report of a cutaneous manifestation of COVID-19. Int J Dermatol. 2020;59(9):1153-1154.

50. Ponce JB, Tjioe KC. Overlapping findings or oral manifestations in new SARS-CoV-2 infection. Oral Dis. 2020;00:1-2. 


\section{Nota conflicto de interés:}

Los autores declaran no tener ningún conflicto de interés.

\section{Nota contribución de los autores:}

1. Concepción y diseño del estudio

2. Adquisición de datos

3. Análisis de datos

4. Discusión de los resultados

5. Redacción del manuscrito

6. Aprobación de la versión final del manuscrito

NSG ha contribuido en 1, 2, 3, 4, 5 y 6.

TNT ha contribuido en $3,4,5$ y 6 .

GVC ha contribuido en 2, 3, 4, 5 y 6 .

AMSR ha contribuido en 3, 4, 5 y 6.

\section{Nota de aceptación:}

Este artículo fue aprobado por la editora de la revista Mag. Dra. Vanesa Pereira-Prado. 